abortions and ectopic pregnancy, and a 33 years old male and 32 years old female worker with primary infertility. The risk assessment has identified all workers were at risk of exposure to hazardous chemicals due to the general ventilation. The chemicals are Chloroform, n-Hexane, Methanol, and Isopropyl alcohol which are known to have evidence of reproductive effect.

Discussion Earlier researches have shows an association between exposure to chloroform and volatile organic compound to infertility and abortion. This study had found the association between exposure to chloroform and VOC with abortion and infertility. There should be more emphasis on the safety and health of workers working with chemicals in the workplace.

\section{EPIDEMIOLOGICAL INVESTIGATION OF A COLLECTIVE CONTAMINATION BY LEAD DURING A RENOVATION OF OLD BUILDINGS, CENTRE REGION, FRANCE, 2010}

${ }^{1} \mathrm{D}$ J Jeannel, ${ }^{2}$ Aymeric, ${ }^{2}$ Laurent, ${ }^{3}$ Baraconnier, ${ }^{3}$ Morel, ${ }^{4}$ Kergresse, ${ }^{5}$ Coly, ${ }^{6}$ Rousseau, ${ }^{2}$ Morvan. 'Orléans, France; ${ }^{2}$ InVS regional office Cire Centre, Orléans, France; ${ }^{3}$ Santé BTP, Blois, France; ${ }^{4} C A P$, Angers, France; ${ }^{5}$ Health agency of Centre region, Orléans, France; ${ }^{6}$ Dirrecte, Orléans, France

\subsection{6/oemed-2013-101717.16}

Following several cases of lead poisoning among building workers in the Centre region (France), the regional office of French Institute for Public Health conducted a retrospective cohort study between May 2008 and December 2009 among the workers involved three renovation projects.

Attack rate for lead contamination $(100 \mathrm{mg} / \mathrm{L} \leq \mathrm{Pb})$ and poisoning $(\mathrm{Pb} \geq 400 \mathrm{mg} / \mathrm{L}$ ) were calculated. An questionnaire was designed to investigate risk factors for lead contamination: 1) exposure to lead on site in relation to work location and duration, compliance with protective measures, 2) other exposure to lead, in professional and personal settings 3 ) knowledge on the risks associated with occupational lead exposureThis survey involved 53 individuals including painters (29\%), roofers (21\%) and electricians (17\%) and others (33\%). Only 11\% were aware of the lead risk on site and 51\% had some knowledge of health hazards related to lead exposure. Of the 44 workers screened for blood lead level, 24 were contaminated, i.e. an attack rate of $54.5 \%$, and 12 were poisoned i.e. an attack rate of $27.2 \%$. Regarding prevention measures, $27 \%$ to $50 \%$ of the cases wore respiratory protection depending on the type of activity and the protection was adapted in about $25 \%$. The risk of contamination/poisoning was associated with: age $(\mathrm{p}=0.003)$, having worked more than a week on site $(R R=5, p=0.003)$ and being directly exposed to lead during their intervention $(\mathrm{RR}=$ $\left.12, \mathrm{p}<10^{-6}\right)$. All intoxicated workers have been directly exposed to lead. This study shows the importance of the health risks associated with lead contamination for workers in old building renovation projects without adequate protection. Poor knowledge of lead-related risks as well as at-risk behaviours were highlighted. Thus, efforts should be made to increase awareness and implement measures to minimise the risks associated with lead exposure.

\section{\begin{tabular}{|l|l}
\hline 17 & LEAD, BLOOD PRESSURE: A CASE-CONTROL STUDY
\end{tabular}}

B Z Boukerma. Université Farhat Abbas, Setif, Algeria

\subsection{6/oemed-2013-101717.17}

In this study, we investigated the relationship between lead and blood pressure of 265 persons (aged 21-59 years) occupationally exposed to inorganic lead ( $\mathrm{PbB}$ between 12.50 and $82.90 \mathrm{mg} / \mathrm{dl}$ ) and 265 people (aged 23 to 59 years) who have ever been exposed to lead (blood lead levels between 0.5 and $5 \mathrm{mg} / \mathrm{dl}$ ). At the time of hiring none of these people had high blood pressure or kidney disease or diabetes. The seniority of these workers varies from 1 year to 35 years. Apart from the basic blood pressure (Bp1: measured at the time of hiring) Blood pressure (Bp2) considered in this study or related to the first measurement of arterial hypertension or to the blood pressure measured in 2010 if the subject is normotensive. The results show that the difference in blood pressure between the 2 groups at the time of hiring (Bp1) is not statistically significant. By cons for the $\mathrm{Bp} 2$ there is a statistically significant difference between the two groups. The study of the survival curves also show a significant difference between the two groups in terms of systolic pressure diastolic.

In the exposed cohort, a multivariate analysis taking into account the covariates and interrelation between the variables shows a an increase in systolic blood pressure and diastolic according of blood lead levels.

In Conclusion Results show that in this study there is a relationship between increased blood pressure and increased levels of blood lead.

\section{Session: C. Health Impact I}

\section{EVIDENCE-BASED CLINICAL QUALITY INDICATORS FOR OCCUPATIONAL HEALTH SERVICES IN THE NATIONAL HEALTH SERVICE (NHS)}

${ }^{1} \mathrm{R}$ R Stilz, ${ }^{2}$ Madan, ${ }^{3} \mathrm{~A}$ Baker. ${ }^{1}$ Plumstead, United Kingdom; ${ }^{2}$ Guy's and St Thomas' NHS Foundation Trust, London, United Kingdom; ${ }^{3} \mathrm{NHS}$-Plus, London, United Kingdom

\subsection{6/oemed-2013-101717.18}

Objectives The use of clinical quality indicators (QI) has been shown to raise standards and reduce variability in clinical care. Clinical QI can also be used for benchmarking and for commissioning services. Few occupational health QI are available. This project developed evidence-based QI for the spectrum of activities carried out by NHS occupational health services.

Methods A systematic literature search was performed, using a two-step hierarchical search strategy. Evidence-based national audits, national guidelines, Cochrane reviews, and systematic reviews were included. Evidence from the literature was translated into QI. Infeasible or irrelevant indicators and indicators based on low level evidence were excluded. Each indicator was assigned a score, reflecting its likely suitability for use in practice.

Results 151 evidence statements were extracted from 44 included publications, resulting in 131 QI. Excluding low grade evidence and irrelevant and infeasible indicators left 65 QI. From these the most suitable 18 QI were developed for activities relating to occupational health clinics, pre-commencement assessments, occupational health monitoring of organisations and occupational health interventions at an organisational level.

Conclusions These 18 QI will populate a new UK based occupational health data registry, the aim of which is to establish an evidence-based quality monitoring system for OHS in the NHS. However, many aspects are also applicable to services outside the NHS and outside the UK. Although these indicators were systematically developed and are based on best available evidence, further work needs to be done to validate theses QI in practice. 